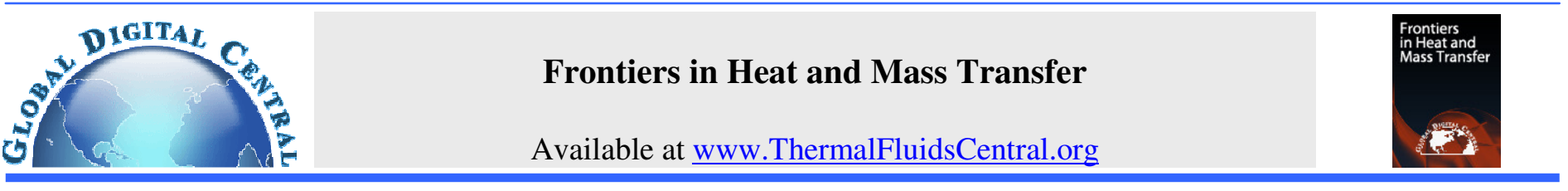

\title{
DETERMINATION OF RADIATION HEAT TRANSFER COEFFICIENT OF STANDING HUMAN BODY MODEL BY NUMERICAL APPROACH
}

\author{
Ahmad Najjaran ${ }^{1}$, Ali R. Tahavvor, Abdolkarim Najjaran, Mohammad A. Ahmadfard \\ Department of Mechanical Engineering, Shiraz Branch, Islamic Azad University, Shiraz, 7154845589, Iran
}

\begin{abstract}
In this paper, external radiation heat transfer coefficient and flux of a standing human body model are calculated for different emissivity coefficients and various temperature differences. To do this, a standing human body sample is designed in such a way that hands and feet are totally open and stretched. Soles are in contact with the ground and so do not have heat transfer. The results are extracted by weighted average method. Despite the existence of the air around the body in reality, the convection heat transfer of human body with the surrounding air is neglected in order to prevent errors and obtain more accurate results. The advantages of the suggested method are able to solve problems with different conditions and obtain more accurate results without the limitations of laboratory experiments.

Keywords: Radiation Coefficient, Radiation flux, Numerical Simulation, Human Body, Emissivity
\end{abstract}

\section{INTRODUCTION}

Radiation is one of the ways of heat transfer that does not need the material environment. Any object with a temperature above absolute zero will emit electromagnetic waves in all directions. Human body exchanges heat with the surrounding in the forms of convection, conduction and radiation. It emits radiation as a black object and therefore for obtaining comfortable environment for occupants, it should be noticed. So, in analyzing and designing of HVAC systems, radiation is one of the factors which should be heeded. In addition, in most countries most of the days are sunny and considering the high temperature of sun, the effects of sun light on human's skin should be noted. Considering to Ozone layer thickness changes, the reflection of radiative heat emitted from sun is varied. These variations sometimes lead to extreme radiative heat which damages skin of humans and sometimes lead to cancers. So radiation analysis of human body is noteworthy in skin health field and illnesses related to, such as skin cancers. Therefore, performing such works seems to need some parameters as radiative coefficient and radiative heat flux of human body at various temperature differences and different emissivity coefficients of the body.

Different works are done related to radiation topics. In some of them convection and radiation are considered for a body and then the heat analysis is done on it (Weng and Chu, 1996). The differences between these researches are the shapes and complexities of the domain, geometry conditions (such as being horizontal or vertical) and boundary conditions. In some works the aim is reviewing the effects of radiation on convection flows (Mohammadein et al., 2008). In some other works it is attempted to find optimal dimensions and geometry for domains to optimize the summation of convection and radiation heat (Khan and Zubair, 1999). Determination of radiation coefficient in different bodies is a general topic of various papers. In some of these papers for determining the radiation coefficient the inverse evaluation method is used. A boundary condition is considered or is determined by some sensors for domain and then by applying an optimization based approach, it is tried to minimize the error difference between the given data and the response from the system (Tadi, 2003). To the best Knowledge of authors, in these researches radiation heat transfer coefficient of human body is determined mostly by experimental research and a few analytical ones (Mochida, 1977) and so no numerical research is done about this issue. As an example, in Ishii (2000) real amounts are used for measuring human body heat exchange. DeDear et al. (1997) and Stolwijk (1970) have used a human body model for measuring heat exchange. Experimental researches have some limitations. For instance, in recent papers body material is selected from different materials such as plastics, while meat and skin are not considered. Ishii (2000), Ichihara et al. (1997), DeDear et al. (1997) and Stolwijk (1970) have reported their information based on considering concrete material for skin of human body. In these papers the human body model is considered in standing and sitting on the chair segments. In most of the researches, the heat radiometer is used for comparing the skin radiation heat and a black object radiation. This performance is because heat transfer coefficients in both conditions are almost equal. Little errors in measurements can change to big errors in heat radiation measurements. Also, it's possible that heat varies in the environment because of different humidity levels. Another difference of this work with the recent ones is that the considered body model is constructed from great numbers of tiny surfaces. These surfaces do not have similar area and shapes. But in recent researches the body is constructed from limited, few numbers of surfaces. Such limitations lead to decreasing the accuracy of calculations and obtaining questionable results.

Some of the researches have been done according to different wave lengths of human skin which are usually supposed to be between 0.95 up to 0.99 (Hendler et al., 1958; Hardy, 1949; Rapp and Gagge , 1967). Body emissivity is estimated by electromagnetic wave reverting principle. Ref (Hendler et al., 1958) also has determined the effect of skin color change on electromagnetic wave reverting approximately $1.7 \%$ for $2 \mathrm{~mm}$ length wave. He has also reported emissivity of human body 0.98. Recently, Ref. (Huang and Togawa, 1995) has presented a non-contact measuring method by camera infrared ray and so he has suggested 0.904 for human body emissivity. This information has been

${ }^{1}$ Corresponding author. Email: anajaran@iaushiraz.ac.ir 
reported for the entire surface of body and not body members separately.

In this work, the radiation heat transfer is simulated by applying the designed model. At first, a standing human body model is designed by computer software. The entire surface of the model is considered in calculations. Surfaces which do not exchange heat with the surrounding are calculated and subtracted. Because of various sizes of remaining approximating surfaces, the calculations of the results are done by weighted average method. Better results are extracted by using this method in comparison with the other averaging methods. A constant average temperature is considered for entire human body sample. Finally, the radiation coefficient and flux of human body skin are calculated using finite volume method. These parameters are reported based on different emissivity coefficients and various temperature differences.

\section{RADIATION OF HUMAN BODY}

In this research, it is assumed that the external skin temperature is constant in the whole body. In addition the body heat transfer with surrounding is assumed steady and in such condition radiation heat transfer whom changes according to skin color, surrounding temperature and body temperature is analyzed.

Thermal radiation of human body plays a great role on his comfort. It should be kept in mind that the body loses radiant heat according to its surface temperature. For a comfortable, normally dressed adult, the weighted average temperature of the bare skin and clothed surfaces is about $(308 \mathrm{~K})$. In still air at a temperature near skin temperature, radiant exchange is the principal form of heat exchange between the body and its environment. If the weighted average of the temperatures of all the surrounding surfaces is $(5 \mathrm{~K})$ hotter or colder than comfortable room air conditions, an occupant will feel uncomfortable. For correcting the situations like this there are some acts which can be done. Firstly, the outside wall surface temperature of building could be changed by adding insulation to the wall construction, or by hanging an insulative tapestry or wall hanging over the wall, as was done in medieval castles. Secondly, the position of the human body might be changed, moving the person closer to an inside wall of building. Thirdly, the temperature of the air might be increased by turning up the thermostat. Increasing the air temperature would decrease the convective heat loss from the body. But if it is supposed that setting the air temperature higher decreases the convective heat loss of body by the same amount that the radiant heat loss would be decreased by moving the body away from the outside wall, maybe everyone else in the room who are not sitting near an outside wall would be too warm. Exactly the same thing might be true during the cooling season.

Human beings as a black body emit radiation in the infrared band. Human body interacts with every other body in a direct line of sight with it. This means that the energy cannot go around corners or be affected by air motion. Radiation is different from convection and conduction. That's why a person near a raging bonfire feels the radiated energy of the fire, even if the surrounding air is very cold. Thermal radiation is generated by the thermal motion of charged particles. Therefore, heat can be transferred through vacuum via radiation versus conduction and convection. In addition, while conduction and convection transfer heat in proportion to the temperature difference between objects, radiation transfers heat in proportion to the fourth powers of the difference between their temperatures. That's why radiative heat transfer to or from human skin is more noticeable when temperature differences of objects are considerable. For example, Measured on an absolute temperature scale, the surface of the sun temperature $(6000 \mathrm{~K})$ is about 20 times that of human body skin $(310$ $\mathrm{K})$. That's why it radiates 160,000 times as much heat toward human body as he radiates toward it. As another example, the dark night sky cools human body quickly because of its extremely low temperature.
Even in cold weather, standing under a leafy tree is much hotter than space.

For determination of the results, different laws are considered in order to minimize the errors and make the results close to the reality. As an example, as expressed by Kirchhoff's law ,the characteristics of thermal radiation depends on various properties of the surface that is emanating form, including its temperature, its spectral absorptivity and spectral emissive power. Plank's law describes the spectrum of black body radiation, which depends only on the object's temperature. In Stefan Boltzmann law the total amount of radiation energy is determined per second (or as power) from a black-body.

$H=\sum_{i=1}^{n} A_{i} e_{i} \sigma T^{4}$

In Eq. (1) $\sigma$ is called the Stefan-Boltzmann constant and is measured for black body. It has a universal value $5.6704 \times 10^{-8}$ $W / m^{2} \cdot K^{4}$. Moreover $\mathrm{A}$ is the cross sectional area, and $\mathrm{T}$ is the absolute temperature in $\mathrm{K}$. As real body is not a perfect black-body radiator, for distinguishing between a perfect black-body and real bodies the idea of emissivity, $e$, has been introduced. A material's ability to absorb and emit thermal radiation is called its emissivity. A perfectly black object has an emissivity of 1 , meaning that it absorbs all thermal radiation that hits it and emits thermal radiation of its own as efficiently as possible. Not all surfaces absorb and emit thermal radiation well. As examples, a highly reflecting or purely white object will have an emissivity close to 0 , meaning that it reflects or scatters almost all the thermal radiation that hits it and does not emit very much thermal radiation of its own. Because most thermal radiation is infrared light, which it cannot be seen; it's not always easy to guess an object's emissivity by looking at it. An object that is white or shiny to visible light may be nearly black to infrared light. Between the human body surface (bare skin and clothing) at average surface temperature $\mathrm{T}$, and the surroundings at temperature $T_{\mathrm{S}}$, the net heat transfer via radiation from the human body to the surroundings is given by the formula (which can be negative if $T<\mathrm{T}_{\mathrm{s}}$ ):

$H_{n e t}=\sum_{i=1}^{n} A_{i} e_{i} \sigma\left(T^{4}-T_{s}^{4}\right)$

The net rate of radiation emitted is the difference between the energy emitted by the human body and the energy absorbs from its surroundings. This net heat transfer is from hot to cold, and it drops to zero when $\mathrm{T}$ becomes equal to $\mathrm{T}_{\mathrm{S}}$. Note that $\mathrm{e}$ is the emissivity of the system, and the emissivity of the surroundings is not involved. (If the surroundings have emissivity less than unity, they will make up the reduced radiation by reflecting back more of the radiation emitted by the system.)

It might be wondering that why the ambient air does not radiate heat toward humans to compensate for the heat they radiate toward it. The answer is that air is reasonably transparent to infrared light, absorbing and emitting relatively little of it. Only water vapor, carbon dioxide, and a few other gases in air interact with infrared light. Thus most of this exchange of energy by radiation is between humans and surrounding surfaces.

In Najjaran et al. (2011) the calculations of radiation coefficient are done with the assumption that the model body is exposed to radiation and convection heat simultaneously. They do this by comparing the effect of radiation and convection. For comparing these parameters linearization law of radiative heat transfer is used. The linearized form of the Eq. (2) looks like Eq. (3).:

$q_{\text {rad }}=h_{r} A\left(T-T_{s}\right)$ 
In Eq. (3)., for obtaining a good approximation $h_{r}$ should be in the following form (Eq. (4)).

$h_{r}=4 \sigma e T_{m}^{3}$

where $T_{m}$ is the arithmetic mean of $T$ and $T_{s}$.

By neglecting conductive heat transfer of human body, heat transfer of human body with surrounding is considered in two types of convection and radiation. Therefore total heat is approximately equal to the summation of radiation and convection heat. Equation (5). shows the general equation of heat transfer.

$$
q_{\text {tot }}^{\prime \prime}=q_{\text {conv }}^{\prime \prime}+q_{\text {rad }}^{\prime \prime}=h_{\text {rad }}\left(T-T_{s}\right)+h_{\text {conv }}(T-T \infty)
$$

The $T_{S}$ is a weighted average of the temperatures of all the surfaces in direct line of sight of the body. Although the $\mathrm{T}_{\mathrm{S}}$ tends to stabilize near the room air temperature, it is also affected by large glass areas, degree of insulation, hot lights, and so on. In this paper it is imagined that $\mathrm{T}_{\mathrm{S}}=\mathrm{T}_{\infty}$. So the Eq. (5) is simplified to

$q_{\text {tot }}^{\prime \prime}=\left(h_{\text {rad }}+h_{\text {conv }}\right)\left(T-T_{\infty}\right)$

\section{FRAMEWORKS}

\subsection{Solution procedure}

At first a sample of human body is designed by SolidWorks ${ }^{\circledR}$ and then its grids are generated by Gambit ${ }^{\circledR}$. The grids are considered unstructured. The final designed model is transferred to Fluent ${ }^{\circledR}$. Boundary conditions (such as body emissivity, body temperature, ambient temperature and pressure) are set as inputs. Against Najjaran et al. research, (2011) the parameters related to convection such as air density are assumed to be zero. The software uses external radiation model and finite volume method for solving the problem. The initial guess is obtained by software and then the desired accuracies of results of governing equations are defined. After convergence of the problem the considered parameters are determined in each grid. Finally, the outputs are extracted by weighted average method.

\subsection{Mathematical definition of weighted average method}

For determining the results several computational methods exist such as actual area average, actual vertex average, standard deviation and the weighted average method (Kurazumi et al., 2008). In this paper weighted average method is used for determining radiation heat transfer coefficient. This method has advantages in the problems which several surfaces with different sizes should be analyzed. The weighted average (or in fact weighted mean) of a non-empty set of radiation coefficients of grids

$\left\{h_{r a d, 1}, h_{r a d, 2}, \ldots, h_{r a d, n}\right\}$

With non-negative weights

$\left\{w_{1}, w_{2}, \ldots, w_{n}\right\}$

is the quantity

$\overline{h_{\text {rad }}}=\left(\sum_{i=1}^{n} w_{i} h_{\text {rad }, i}\right) /\left(\sum_{i=1}^{n} w_{i}\right)$

Therefore, radiation coefficients of grids with a high weight contribute more to the weighted average than do radiation coefficients of grids with a low weight. The weights cannot be negative. Some may be zero, but not all of them because division by zero is not allowed. The formulas are simplified when the weights are normalized such that they sum up to 1, i.e. $\sum_{i=1}^{n} w_{i}=1$. For such normalized weights the weighted average is simply $\overline{h_{\text {rad }}}=\sum_{i=1}^{n} w_{i} h_{r a d, i}$.

\section{RESULT AND DISCUSSION}

In previous papers, plastic or concrete mannequins were used because of experimental limitations. In addition, in some works body surface is divided to a few limited parts and for each part a specific temperature is assumed and a radiation coefficient is obtained. In these papers this assumption is used because the temperature gradient varies on skin surface caused by the changes of blood circulations and other factors.

In this paper skin and meat are considered for materials of human body. Heat transfer is considered steady and the surrounding is assumed uniform and stable. As the assumption of various constant temperatures for different parts of human body leads to lower results accuracy; in Kurazumi et al. research (2008), measurements are done by assuming constant temperature for whole body and this temperature is obtained by the average temperature of different parts of body. In this paper, this assumption is used and an average temperature $(308 K)$ is applied for the whole body. In addition, the radiation coefficients are calculated for the entire body and not several parts of the body. The temperature of environment is assumed constant $293 \mathrm{~K}$. These assumptions are useful in gaining better results and causes simulation to be close to reality.

One of the differences between the present work and the previous ones is that the body is organized from a large number of approximating surfaces. The standing human body model is constructed from 1874427 approximating surfaces, 191524 boundary nodes and 896726 cells. The shape of grids is tetrahedron. These quantities show the complexity of the problem. Body grid simulation has been done in such a way that different parts of body have no contact with each other (see Fig. 1.). For example, fingers have no contact with each other. The accuracy of constructed mesh grids are checked in different directions by some sectional planes. As is shown in Fig. 2 the grid concentration is more around the body. This causes more accurate results and decreases the grid numbers. The reasons of the excess number of the human body grids and concentration of grids around the body are the complex structure of body and conjugation of thermal heat exchange around the body.

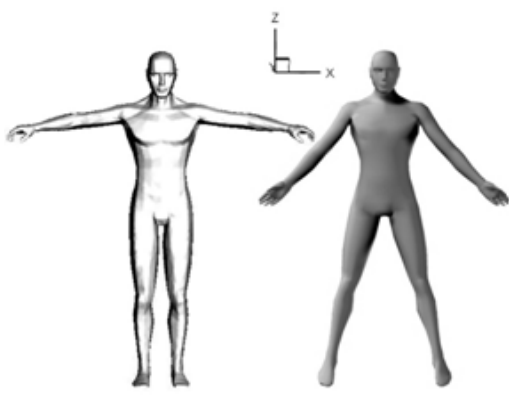

Fig. 1 Considered standing human body models

A domain is considered around the body. The considered domain is limited only from the ground and it does not have any limitation (such as walls) from the other sides. The ground and ambient are considered in a thermal balance and with the same temperatures. Figure 3. shows a sample of domain around body model. As mentioned before in Najjaran 
et al. (2011) because of temperature differences between body and surrounding, some heat is supposed to transfer to environment in convectional form. This heat causes the temperature gradient in surrounding air and this gradient leads to density gradient. Considering the existence of buoyancy force, the surrounding fluid flows. But as the convection is irrelevant to radiation and existence of it around the body conjugates the problem and leads to generating errors; so in this paper the convectional heat is neglected and the calculations are based on the radiation heat $\mathrm{q}_{\text {rad }}^{\prime \prime}$ and not total heat against Najjaran et al. (2011). By using Fluent and applying weighted average method the unknowns are determined.

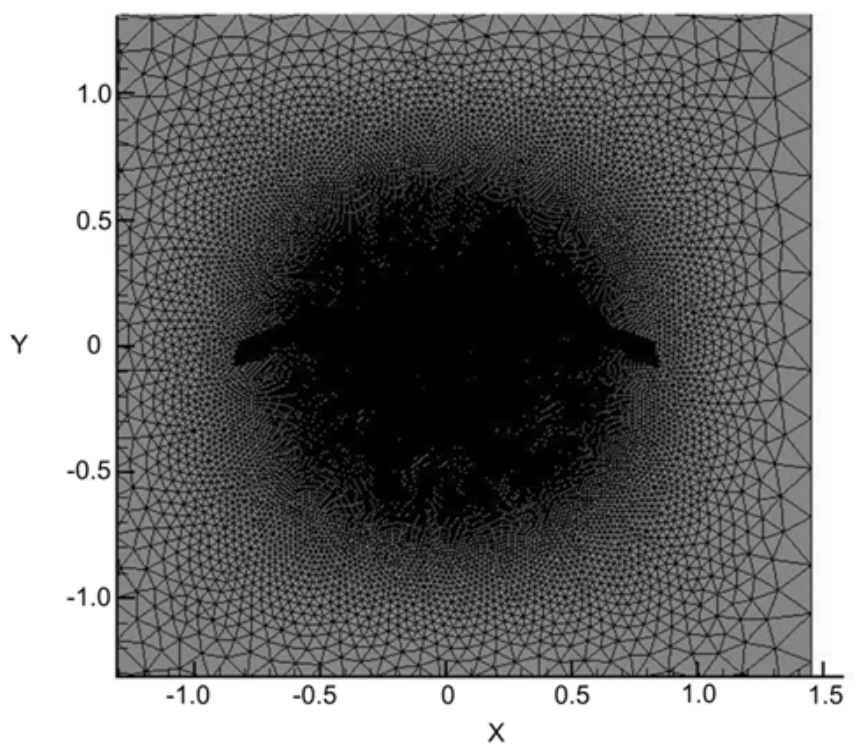

Fig. 2 Mesh gird around human body model

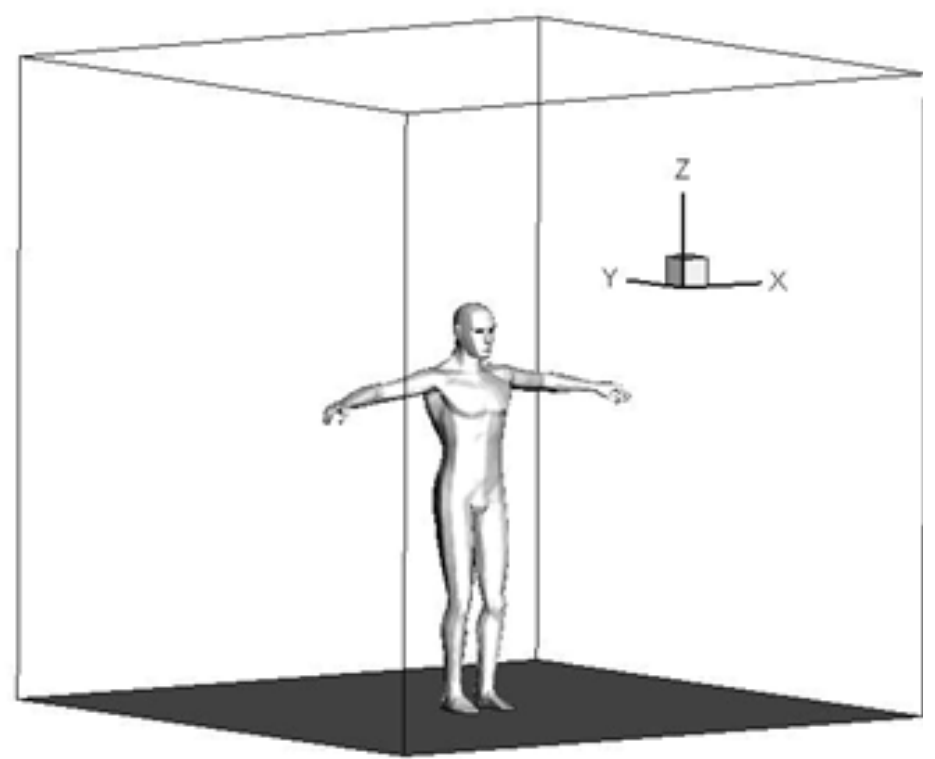

Fig. 3 Considered domain around human body model

Table 2 shows the radiation coefficients which are reported in Najjaran et al. (2011). It can obviously be seen that the coefficients are a bit less than those which are obtained in this work in temperature difference of $15 \mathrm{~K}$.

The standing human body model is studied in 36 different cases. In these cases the radiation coefficient and the radiation flux of body are obtained based on different emissivity coefficients and various temperature differences. The radiation coefficients and heat fluxes which are reported in Table 1 are reported in $\mathrm{W} / \mathrm{m}^{2} \mathrm{~K}$ and $\mathrm{W} / \mathrm{m}^{2}$, respectively.

Table 1 Radiation coefficients and radiation heat fluxes related to emissivity and temperature differences for standing human body model Emissivity

\begin{tabular}{rrrrrrr} 
& \multicolumn{7}{c}{0.95} & 0.96 & 0.97 & 0.98 & 0.99 & 1.0 \\
\cline { 2 - 7 }$\Delta T$ & \multicolumn{5}{c}{$\mathrm{h}$} \\
\cline { 2 - 7 } 19 & 3.75 & 3.8 & 3.8 & 3.87 & 3.87 & 3.88 \\
17 & 3.77 & 3.83 & 3.84 & 3.89 & 3.94 & 3.99 \\
15 & 3.79 & 3.85 & 3.86 & 3.91 & 3.96 & 4.01 \\
13 & 3.84 & 3.87 & 3.88 & 3.97 & 3.99 & 4.02 \\
11 & 3.88 & 3.94 & 3.94 & 4.05 & 4.13 & 4.21 \\
9 & 3.91 & 4.02 & 4.14 & 4.25 & 4.37 & 4.48 \\
\cline { 2 - 7 }
\end{tabular}

\begin{tabular}{cccccc}
\hline 0.95 & 0.96 & 0.97 & 0.98 & 0.99 & 1.0 \\
\hline 71.19 & 72.16 & 72.22 & 73.57 & 73.6 & 73.62 \\
64.03 & 65.07 & 65.35 & 66.17 & 67.0 & 67.82 \\
56.86 & 57.75 & 57.93 & 58.65 & 59.36 & 60.07 \\
49.86 & 50.25 & 50.5 & 51.61 & 51.97 & 52.32 \\
42.61 & 43.31 & 43.34 & 44.58 & 45.45 & 46.31 \\
35.18 & 36.21 & 37.23 & 38.25 & 39.3 & 40.3 \\
\hline
\end{tabular}

Table 2 Radiation coefficients and radiation heat fluxes related to emissivity and temperature differences for standing human body model reported in Najjaran et al. (2011)

\section{Emissivity}

\begin{tabular}{rrrrrrr}
\cline { 2 - 6 }$\Delta T$ & 0.95 & 0.96 & 0.97 & 0.98 & 0.99 & 1.0 \\
\cline { 2 - 7 } 15 & 3.79 & 3.806 & 3.834 & 3.865 & 3.88 & 3.937 \\
\cline { 2 - 7 }
\end{tabular}

Emissivity

\begin{tabular}{cccccc}
\hline 0.95 & 0.96 & 0.97 & 0.98 & 0.99 & 1.0 \\
\hline \multicolumn{7}{c}{$\mathrm{q}$} \\
\hline 59.06 & 58.2 & 57.98 & 57.52 & 57.10 & 56.85 \\
\hline
\end{tabular}


By curve fitting the data which are expressed in Table 1, Figs 4 to 6 will be obtained. Figure 4. illustrates the relationship of radiation coefficient with emissivity and temperature differences for standing segment.

This diagram has a stead falling trend. Regular trend exists between 9-11 degrees of temperature in a form that $\mathrm{e}=1$ has the most and $\mathrm{e}=0.95$ has the least amounts.

Equation (8) shows the function of curves which are illustrated in Fig. 4 and Table 3 illustrates the parameters of each curve in this equation.

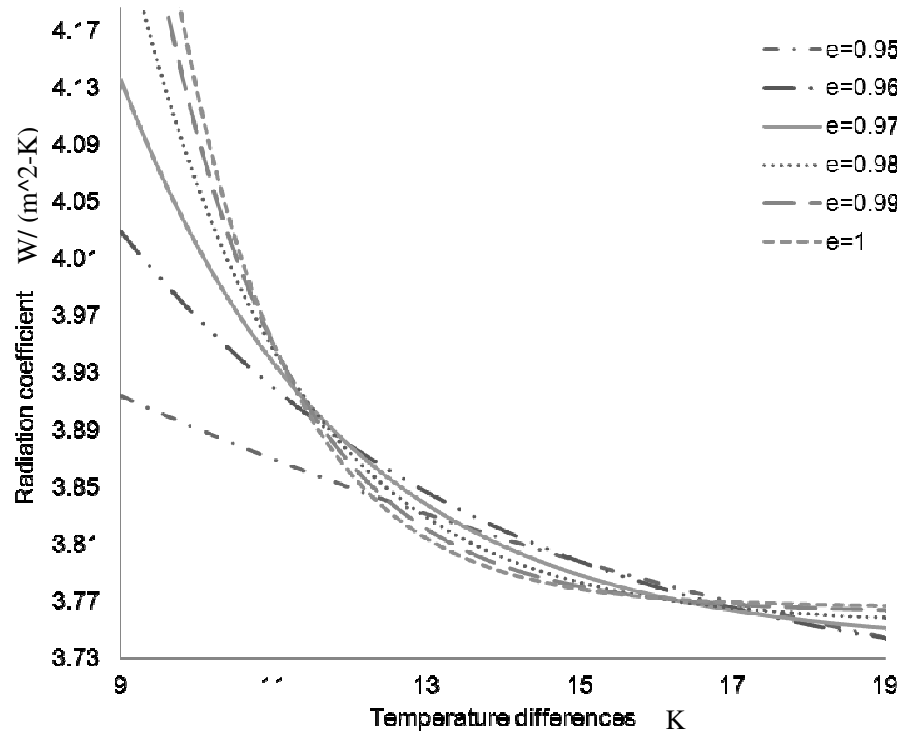

Fig. 4 Relationship of radiation coefficient with emissivity (e) and temperature differences for standing segment

$h_{r}=a+b \exp (-T / c)$

Figure 5 also shows the relationship of radiation flux with emissivity (e) and radiation coefficients.
Equation (9) shows the function of curves which are illustrated in Fig.5 and Table 4 illustrates the parameters of each curve in this equation.

$q=a+b \exp \left(-h_{r} / c\right)$

Table 3 The parameters of Eq. 8 related to curves in Fig. 4

\begin{tabular}{cccc}
\hline $\mathbf{e}$ & $\mathbf{a}$ & $\mathbf{b}$ & $\mathbf{c}$ \\
\hline $\mathbf{9 5}$ & 3.588 & 0.631 & 13.626 \\
$\mathbf{9 6}$ & 3.701 & 2.051 & 4.913 \\
$\mathbf{9 7}$ & 3.739 & 9.085 & 2.875 \\
$\mathbf{9 8}$ & 3.754 & 34.893 & 2.115 \\
$\mathbf{9 9}$ & 3.762 & 114.172 & 1.716 \\
$\mathbf{1 0 0}$ & 3.766 & 305.494 & 1.484 \\
\hline
\end{tabular}

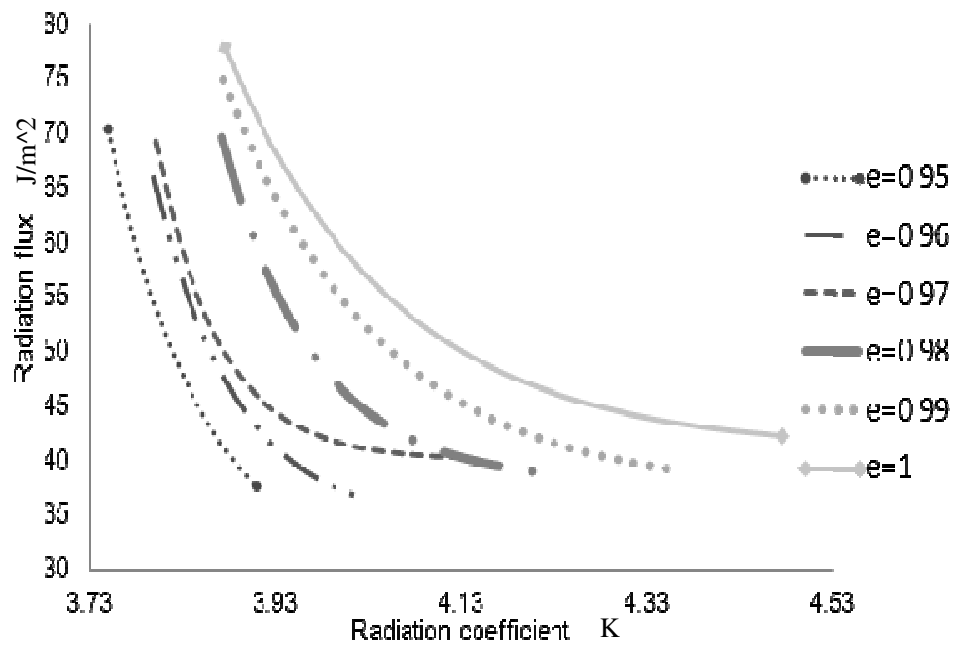

Fig. 5 The relationship of radiation flux with emissivity (e) and radiation coefficien

Table 4 The parameters of Eq. 9 related to curves in Fig. 5

\begin{tabular}{cccc}
\hline $\mathbf{e}$ & $\mathbf{a}$ & $\mathbf{b}$ & $\mathbf{c}$ \\
\hline $\mathbf{9 5}$ & 29.006 & $2.66 \times 10^{17}$ & 0.103 \\
$\mathbf{9 6}$ & 34.075 & $1.78 \times 10^{20}$ & 0.088 \\
$\mathbf{9 7}$ & 40.057 & $5.52 \times 10^{25}$ & 0.068 \\
$\mathbf{9 8}$ & 37.949 & $3.07 \times 10^{18}$ & 0.099 \\
$\mathbf{9 9}$ & 37.069 & $3.91 \times 10^{11}$ & 0.168 \\
$\mathbf{1 0 0}$ & 40.936 & $6.52 \times 10^{10}$ & 0.182
\end{tabular}

The relation of radiation flux with temperature differences and emissivity coefficients are shown schematically in Fig. 6.

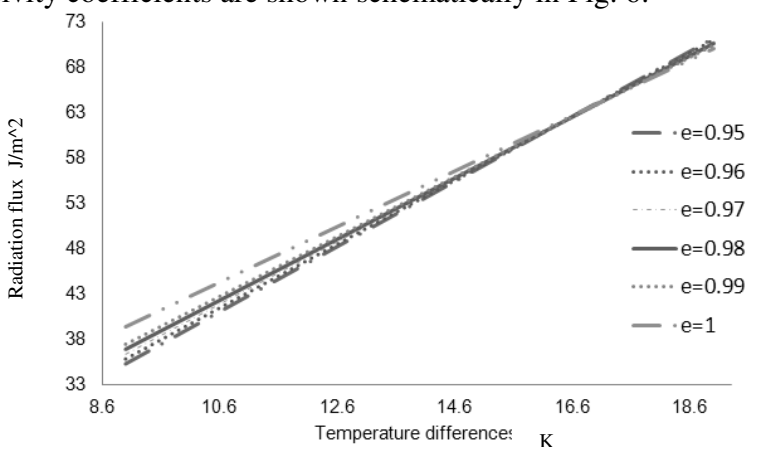

Fig. 6 The relation of radiation flux with temperature differences and emissivity coefficients
Equation (10) and Table 5 describe function and parameters of each curve respectively.

$$
q=a+b T
$$

Table 5 The parameters of Eq. 10 related to curves in Fig. 6

\begin{tabular}{ccc}
\hline $\mathbf{e}$ & $\mathbf{a}$ & $\mathbf{b}$ \\
\hline $\mathbf{9 5}$ & 3.026 & 3.59 \\
$\mathbf{9 6}$ & 4.219 & 3.517 \\
$\mathbf{9 7}$ & 5.41 & 3.444 \\
$\mathbf{9 8}$ & 6.604 & 3.371 \\
$\mathbf{9 9}$ & 7.829 & 3.296 \\
$\mathbf{1 0 0}$ & 11.827 & 3.066 \\
\hline
\end{tabular}




\section{CONCLUSIONS}

The aim of this research is determining the radiation coefficient and radiation flux of human body based on various temperature differences and different emissivity coefficient by numerical simulation. To do this, radiation of a standing human body model was simulated. The results are calculated by applying finite volume method and extracted by weighted average method. The parameters are calculated for the entire body. The ambient and body temperatures are considered constant. Heat transfer of body is assumed steady and the ambient assumed uniform. The radiation heat transfer was in a form of external radiation. Several conditions are considered and for each one the problem is solved and finally the achieved results are reported. The results show the ability of suggesting method to solve various types of problems with different conditions related to radiation of human body.

\section{NOMENCLATURE}

$\begin{array}{ll}\mathrm{A} & \text { Surface area }\left(\mathrm{m}^{2}\right) \\ \mathrm{T} & \text { Absolute temperature }(\mathrm{K}) \\ \mathrm{q}_{\text {conv }}^{\prime \prime} & \text { Convection heat flux }\left(\mathrm{W} / \mathrm{m}^{2}\right) \\ \mathrm{q}_{\text {rad }}^{\prime \prime} & \text { Radiation heat flux }\left(\mathrm{W} / \mathrm{m}^{2}\right) \\ \mathrm{q}_{\text {tot }}^{\prime \prime} & \text { Total heat flux }\left(\mathrm{W} / \mathrm{m}^{2}\right) \\ \mathrm{h}_{\text {conv }} & \text { Convection coefficient }\left(\mathrm{W} / \mathrm{m}^{2} \mathrm{~K}\right) \\ \mathrm{h}_{\mathrm{r}} & \text { Radiation coefficient }\left(\mathrm{W} / \mathrm{m}^{2} \mathrm{~K}\right) \\ \mathrm{q}_{\text {rad }} & \text { Radiation heat }(\mathrm{W}) \\ \mathrm{T}_{\infty} & \text { Temperature of surrounding }(\mathrm{K})\end{array}$

Greek Symbols

$\varepsilon \quad$ Gray body emissivity of the object

$\sigma \quad$ Stefan-Boltzmann constant $5.67 \times 10^{-8}\left(\mathrm{~W} / \mathrm{m}^{2} \mathrm{~K}^{4}\right)$

$\infty \quad$ ambient environment

\section{REFERENCES}

DeDear, R.J., Arens, E., Hui, Z., Oguro, M., 1997, "Convective And Radiative Heat Transfer Coefficients For Individual Human Body Segments" International Journal of Biometeorology, 40(1), 41-56.

Hardy, J.D., 1949, Heat transfer. In: Newburgh LH (ed) Physiology of heat regulation and the science of clothing, Philadelphia, Saunders, pp 78-108.

Hendler, E., Crosbie, R., Hardy, J.D., 1958, "Measurement of Heating of the Skin during Exposure to Infrared Radiation" Journal of Applied Physiology, 12(1), 77-85.

Huang, J., Togawa, T., 1995, "Improvement of Imaging of Skin Thermal Properties by Successive Thermographic Measurements at a
Stepwise Change in Ambient Radiation Temperature" Physiological Measurement 16(2), 95-301.

http://dx.doi.org/10.1088/0967-3334/16/4/009

Ichihara, M., Saitou, M., Nishimura, M., Tanabe, S., 1997, "Measurement Of Convective And Radiative Heat Transfer Coefficients Of Standing And Sitting Human Body By Using A Thermal Manikin" Journal of Architecture, Planning and Environmental Engineering (Transactions of AIJ), 50(1), 45-51.

Ishii, J., 2000, "The Combined Effect of Operative Temperature, Illuminance and Light Source on the Human Physiological and Psychological responses In Indoor Environment" Doctoral thesis, Nagoya Institute of Technology, pp. 37-68.

Khan, J., Zubair, S.M., 1999, "The optimal dimensions of convectiveradiating circular fins" Heat and Mass Transfer, 35, 469-478. http://dx.doi.org/10.1007/s002310050350

Kurazumi, Y., Tsuchikawa, T., Ishiia, J., Fukagawaa, K., Yamatoc, Y., Matsubarad, N., 2008, "Radiative and Convective Heat Transfer Coefficients of The Human Body in Natural Convection," Building and Environment, 43, 2142-2153.

http://dx.doi.org/10.1016/j.buildenv.2007.12.012

Mochida, T., 1977, "Convective and Radiative Heat Transfer Coefficients for Human Body" Transactions of AIJ, 258, 63-9.

Mohammadein, A.A., Aissa, W.A., Rama, S.R.G., 2008, "The effect of radiation on mixed convection flow past a stretching surface" Heat and Mass Transfer, 44, 1035-1040.

http://dx.doi.org/10.1007/s00231-007-0334-8

Najjaran, A., Tahavvor A.R., Najjaran Ak., Ahmadfard M.A., 2011, "Numerical Simulation of Radiation Heat Transfer of the Human Body for Determining Its Radiation Coefficient," ASME International Mechanical Engineering Congress \& Exposition, November 11-17, Denver, Colorado, USA. http://dx.doi.org/10.1115/IMECE2011-65353

Rapp, G.M., Gagge, A.P., 1967, "Configuration Factors and Comfort Design In Radiant Beam Heating Of Man By High Temperature Infrared Sources" ASHRAE Transactions 73(II), III.1.1-III.1.18.

Stolwijk, J.A.J., 1970, "Mathematical Model of Thermoregulation. In: Hardy JD, Gagge AP, Stolwijk JAJ (eds) Physiological and behavioral temperature regulation," Springfield Thomas, pp 703-21.

Tadi, M., 2003, "Inverse evaluation of emissivity function," Heat and Mass Transfer, 40, 15-23. http://dx.doi.org/10.1007/s00231-002-0379-7

Weng, L.C., Chu, H.S., 1996, "Combined natural convection and radiation in a vertical annulus," Heat and Mass Transfer, 31(3), 71-37. http://dx.doi.org/10.1007/s002310050070 